Sensitivity matrix and reconstruction algorithm for EIT assuming axial uniformity

Jerbi, Karim and Lionheart, William and Vauhkonen, Paivi and Vauhkonen, Marko

2013

MIMS EPrint: 2013.54

Manchester Institute for Mathematical Sciences

School of Mathematics

The University of Manchester

\footnotetext{
Reports available from: http://eprints.maths.manchester.ac.uk/

And by contacting: The MIMS Secretary

School of Mathematics

The University of Manchester

Manchester, M13 9PL, UK
} 


\title{
Sensitivity Matrix and Reconstruction Algorithm for EIT Assuming Axial Uniformity
}

\author{
K. Jerbi ${ }^{1}$, W.R.B. Lionheart ${ }^{2}$, P.J. Vauhkonen ${ }^{3}$, M. Vauhkonen ${ }^{4}$ \\ 1 Biomedical Engineering Institute, University of Karlsruhe, Germany, up0c@rz.uni- \\ karlsruhe.de \\ 2 Department of Mathematics, University of Manchester, UK, bill.lionheart@umist.ac.uk \\ 3 Department of Applied Physics, University of Kuopio, Finland, pvauhkonen@messi.uku.fi \\ 4 Department of Applied Physics, University of Kuopio, Finland, mvauhkonen@messi.uku.fi
}


This is a preprint. Now published as Physiol. Meas. 21 (2000) 61-66.

Abstract - In Electrical Impedance Tomography (EIT) two-dimensional models continue to be applied despite their known inability to provide correct reconstruction. In this paper, a reconstruction algorithm that assumes a translationally invariant conductivity distribution is described. A more precise forward solver is obtained by taking off-slice currents into consideration. An appropriate sensitivity matrix is derived. Numerical evidence for the improvement in precision compared to two-dimensional reconstruction is given. 


\section{INTRODUCTION}

Electrical impedance tomography which poses the problem of reconstructing the conductivity distribution inside a region from electrical measurements made on the boundary is clearly a three-dimensional problem. The algorithms associated with two-dimensional models assume that the current injected into a region is restricted to the measurement plane i.e. the plane determined by the electrodes used for voltage measurements. This assumption which is obviously false leads to incorrect reconstruction. Correct boundary shape but also a model consistent with the dimension of the problem are essential for static image reconstruction in EIT [3]. The known drawbacks of 3D finite element routines are excessive storage requirements but above all lengthy execution times caused by the huge number of unknowns. In this study we use a threedimensional model which assumes that the conductivity distribution is invariant in the third (axial) dimension. We derive an appropriate sensitivity matrix using the perturbation method and a linearisation of the posed problem. Numerical results are given and the ill-posedness of the problem is discussed.

\section{GENERAL 3D FORMULATION}

For an isotropic and linear medium the law of conservation of charge in the case of steady currents is expressed as

$$
\nabla \cdot(\sigma \vec{E})=0
$$

where $\vec{E}$ is the electric field and $\sigma(x, y, z)$ is the conductivity distribution. The electric field is in this case conservative. It can therefore be expressed in terms of an electric potential $\phi(x, y, z)$ as follows:

$$
\vec{E}=-\nabla \phi(x, y, z)
$$

It is easy to see that inserting (2) in (1) yields the governing equation for three-dimensional EIT:

$$
\nabla \cdot \sigma(x, y, z) \nabla \phi(x, y, z)=0
$$

Applying current patterns at the boundary by means of current injecting electrodes leads to boundary conditions given by:

$$
\sigma \frac{\partial \phi}{\partial n}=\left\{\begin{array}{cc}
J & \text { on current electrodes } \\
0 & \text { elsewhere on the surface }
\end{array}\right.
$$

where $\frac{\partial \phi}{\partial n}$ is the gradient of the potential distribution normal to the boundary and $J$ is the current density on the surfaces of the electrodes. The inverse problem of EIT consists in solving the above defined boundary value problem in three dimensions. The solution for such problems is known to be unique.

\section{RESTRICTION TO THE CASE OF TRANSLATIONALLY INVARIANT CONDUCTIVITIES}

In this paragraph we will consider the special case of a constant conductivity distribution along the z-axis within a three-dimensional domain of height $2 a$ (Fig.1). 


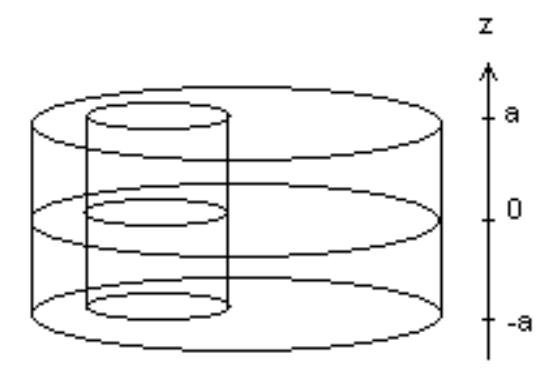

Figure 1: A 3-D domain of height 2a with translationally invariant conductivities.

The potential distribution function $\phi(x, y, z)$ is obviously symmetric to the current injection plane $z=0$. The Fourier expansion of the potential is thus given by the following cosine series (Ider et al., 1990):

$$
\phi(x, y, z)=\sum_{k=0}^{\infty} V_{k}(x, y) \cos \left(\frac{k \pi}{a} z\right)
$$

where $V_{k}(x, y)$ is the two-dimensional potential for the frequency $k$. Substituting Eq. 5 into the governing equation for the general three dimensional case results in the following twodimensional partial differential equations (PDE):

$$
\nabla \cdot \sigma \nabla V_{k}-\sigma\left(\frac{k \pi}{a}\right)^{2} V_{k}=0
$$

The typical Laplacian equation in 3-D is thereby split into an infinite number of 2-D PDE equations. The corresponding boundary conditions are given by

$$
\sigma(x, y) \frac{\partial V_{k}}{\partial n}=J_{k}
$$

where $J_{k}$ is the $k$ th term in the cosine expansion of the boundary conditions. Truncation of the cosine series allows quick reconstruction with negligible loss in precision. In [1] and [2] ten and eight terms of the series were respectively reported to be sufficient to describe the solution.

\subsection{Finite Element forward solver}

The Galerkin method leads to a system stiffness matrix for each frequency term $k$ of the cosine expansion.

For every element of the two-dimensional mesh generated for the measurement plane the element stiffness matrix is defined by

$$
a_{i j}^{e}=\int_{\Omega} \nabla N_{i} \cdot \nabla N_{j}+\left(\frac{k \pi}{a}\right)^{2} N_{i} N_{j} d \Omega
$$

where $N_{i}$ and $N_{j}$ are the shape functions. Note that the conductivity has been omitted due to the assumption of a piecewise constant conductivity distribution. The forcing vector i.e. the so-called right hand side vector for each term $k$ is given by

$$
\int_{\Gamma} N_{i} J_{k} d \Gamma
$$

where $\Gamma=\partial \Omega$ and $J_{k}$ is the $k$ th Fourier coefficients of the current density $J$ such that, 


$$
J_{0}=2 \frac{h}{H} J
$$

and

$$
J_{k}=\frac{2}{k \pi} J \sin \left(\frac{k \pi h}{H}\right)
$$

where $H=2 a$ is the total height of the domain and $\mathrm{h}$ is the electrode height satisfying $h<H$. Compared to the two-dimensional algorithm this algorithm requires three extra parameters: the maximum frequency term $k_{\max }$, the total domain height $H$ and the electrode height $h$.

\subsection{Sensitivity Matrix ( Linearisation )}

The inverse problem of EIT consists in deducing the conductivity distribution from the voltage measurements on the boundary. Reconstruction has been attempted for the special case of a separable potential distribution described above using the Iterative Equipotential Line Method (IELM) which is based on the computation of a two-dimensional equivalent form of the measurements using a scaling method that relies on the calculation of a 2D/3D ratio for a homogeneous conductivity distribution. The search for the conductivity distribution is then carried out using the so-called two-dimensional equivalent form of the measurements. Several fundamental limitations of the IELM are reported in [1]. In this study we derive a sensitivity matrix based on the perturbation method. A perturbation of the conductivity $\sigma+\delta \sigma$ under constant boundary conditions is associated with a perturbation of boundary voltages $u+\delta u$. A conductivity update could therefore be determined from boundary voltage differences between the measured potentials obtained from the tomograph and the calculated potentials given by the forward model. The sensitivity matrix (see appendix) in integral form for a given frequency term $k$ is given by

$$
\int_{\Gamma} \delta \mathrm{u}_{\kappa} \sigma \nabla_{\mathrm{n}} \omega \delta \Gamma=-\int_{\Omega} \delta \sigma\left(\nabla \omega \cdot \nabla \mathrm{u}_{\kappa}+\left(\frac{\kappa \pi}{\alpha}\right)^{2} \omega \mathrm{u}_{\kappa}\right) \delta \Omega
$$

where $\nabla_{\mathrm{n}}$ denotes the normal derivative and $\omega$ is any weighting function that satisfies the governing PDE

$$
\nabla \cdot \sigma \nabla \omega-\sigma\left(\frac{k \pi}{a}\right)^{2} \sigma \omega=0
$$

The overall sensitivity matrix is obtained by adding up the sensitivity matrices calculated for each frequency $k$ term of the cosine series.

\section{FORWARD SOLVER EVALUATION}

To investigate the precision of the above defined forward solver and to compare its performance to that of a two-dimensional algorithm the reconstruction of known homogeneous conductivity distributions was attempted. A reconstruction problem given a homogeneous conductivity distribution is governed by the Laplace's equation

$$
\sigma_{\text {hom }} \Delta \phi=0
$$

Starting with an initial conductivity value set to the unit i.e. $\sigma_{0}=1 \Omega^{-1} \mathrm{~m}^{-1}$ the calculation of the actual conductivity value in the case of a correct forward model is equivalent to the calculation of the scaling factor which minimizes the $L^{2}$ norm of the error functional expressing the difference between measured and calculated boundary voltages. To this purpose simulation data of cylindrical domains were used. The data were generated by a three-dimensional finite element 
model simulating three tanks with a given conductivity $\sigma_{\text {hom }}$, cylinder height $H$ and electrode height $h$ (Table 1). Tables 2,3 and 4 show the result of the reconstruction for each data set in the classical two-dimensional case (2-D) and for the separable case (21/2-D) for which the cosine series was truncated after the $10^{\text {th }}$ term. Notice that for these reconstructions the first 6 trigonometric current injection patterns were used.

\begin{tabular}{|c|c|c|c|}
\hline & Tank 1 & Tank 2 & Tank 3 \\
\hline $\mathrm{h}[\mathrm{cm}]$ & 1.5 & 1.0 & 1.0 \\
\hline $\mathrm{H}[\mathrm{cm}]$ & 3 & 5 & 7 \\
\hline$\sigma_{\text {hom }}[\mathrm{mS} / \mathrm{cm}]$ & 2.5 & 10 & 1.25 \\
\hline \multicolumn{4}{|c}{ Table 1 }
\end{tabular}

\begin{tabular}{|c|c|c|c|}
\hline Tank 1 & $\sigma_{\text {hom }}$ & $\sigma_{\text {cal }}$ & $\varepsilon_{\mathrm{r}}$ \\
\hline 2-D & 2.5 & 5.3 & 1.12 \\
\hline 21/2-D & 2.5 & 2.7 & 0.08 \\
\hline \multicolumn{3}{|c|}{ Table 2 }
\end{tabular}

\begin{tabular}{|c|c|c|c|}
\hline Tank 2 & $\sigma_{\text {hom }}$ & $\sigma_{\text {cal }}$ & $\varepsilon_{\mathrm{r}}$ \\
\hline 2-D & 10.0 & 48.3 & 3.83 \\
\hline 21/2-D & 10.0 & 09.7 & 0.03 \\
\hline \multicolumn{4}{|c|}{ Table 3 }
\end{tabular}

\begin{tabular}{|c|c|c|c|}
\hline Tank 3 & $\sigma_{\text {hom }}$ & $\sigma_{\text {cal }}$ & $\varepsilon_{\mathrm{r}}$ \\
\hline 2-D & 1.25 & 7.78 & 5.23 \\
\hline 21/2-D & 1.25 & 1.19 & 0.04 \\
\hline \multicolumn{4}{|c|}{ Table 4 } \\
\hline
\end{tabular}

Looking at the relative error it becomes clear that the solver based on a three-dimensional model assuming uniform conductivity in the axial direction leads to a clear improvement in precision when compared to the classical two-dimensional case.

\section{CONCLUSIONS AND DISCUSSION}

Given translational uniformity the above algorithm provides a more precise forward solver which takes the third dimension of the problem into consideration. As a result the sensitivity matrix we derive to solve the inverse problem is also better suited. Image reconstructions have been successfully achieved using this algorithm with both simulation and In-vivo thoracic data. Little change was noticed for the conductivity distribution when the reconstructed conductivity images were compared to those obtained from the classical two-dimensional solver. However, the static conductivity values showed a definite improvement in precision. In the case of simulations of a tank containing 2 phantoms (two cylinders one of higher and one of lower conductivity than the background) the reconstructed values were clearly closer to the actual conductivities used to produce the data. The In-vivo thoracic data also yielded conductivity values which came much closer to the expected physiological values. However, it is important to keep in mind that the obtained values depend upon the choice of the parameter $\mathrm{H}$ (i.e. the total height of the body assumed to be axially invariant with respect to conductivity). It is therefore necessary to give special attention to the choice of this parameter. For bodies with variable conductivity in the axial direction (e.g. human thorax) a rule of thumb could be developed based on experimental data. The degree of ill-posedness was investigated by singular value decomposition of the derivative (sensitivity) matrix. The condition number which is given by $\alpha=\lambda_{\max } / \lambda_{\min }$ where $\lambda_{i}$ denote the singular values has the same order of magnitude as the condition number calculated for the twodimensional case (see fig. 2). Hence, the problem seems to be ill-posed in a similar way. Finally, it can be concluded that the proposed approach is definitely a better choice than the genuine two-dimensional approach for static imaging. Furthermore, its high speed (comparable to 2D) makes it an attractive alternative to three-dimensional solvers given a tolerably small variation of the conductivity distribution along the third axis. In industrial Process Tomography the translationally uniform assumption will be valid in some cases involving for example cylindrical pipes. In the medical field limbs come closest to translational invariance. For thoracic imaging clearly three-dimensional forward models and reconstruction are needed. However, when only two-dimensional data are available the translationally invariant approach may prove a worthwhile improvement over purely two-dimensional imaging. 


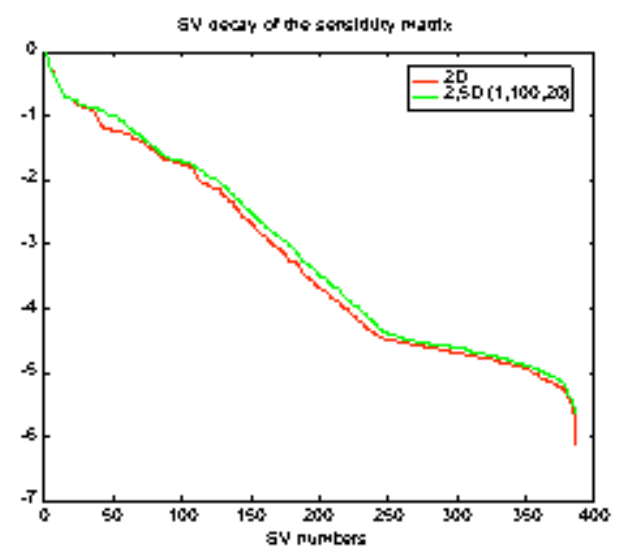

Figure 2: Singular value decay of the sensitivity matrices for a body shape mesh with a homogeneous conductivity. The upper trace represents the translationally uniform case and the lower the 2-D case.

\section{ACKNOWLEDGEMENTS}

The authors wish to thank Prof. O. Doessel of the Biomedical Engineering Institute at the University of Karlsruhe, Germany and Dr. C. Mcleod from the School of Engineering at the Oxford Brookes University, U.K. for their valuable help and support. This work is partially supported by the Wellcome Trust.

\section{APPENDIX}

\section{Sensitivity Matrix:}

Consider the weak formulation for the perturbation:

$\int_{\Omega} \omega\left[\nabla \cdot(\sigma+\delta \sigma) \nabla\left(\mathrm{u}_{\mathrm{k}}+\delta \mathrm{u}_{\mathrm{k}}\right)-\left(\frac{\pi \mathrm{k}}{\mathrm{a}}\right)^{2}(\sigma+\delta \sigma)\left(\mathrm{u}_{\mathrm{k}}+\delta \mathrm{u}_{\mathrm{k}}\right)\right] \mathrm{d} \Omega=0$

where $\omega$ satisfies the following PDE:

$\nabla \cdot \sigma \nabla \omega-\left(\frac{\pi \mathrm{k}}{\mathrm{a}}\right)^{2} \sigma \omega=0$

Applying the governing equation for $\sigma$ and $u_{k}$ and neglecting higher order terms gives:

$\int_{\Omega} \omega\left[\nabla \cdot \sigma \nabla \delta \mathrm{u}_{\mathrm{k}}+\nabla \cdot \mathrm{u}_{\mathrm{k}} \nabla \delta \sigma-\left(\frac{\pi \mathrm{k}}{\mathrm{a}}\right)^{2}\left(\sigma \delta \mathrm{u}_{\mathrm{k}}+\delta \sigma \mathrm{u}_{\mathrm{k}}\right)\right] \mathrm{d} \Omega=0$

Using Green's laws and constant boundary conditions $\delta\left(\sigma \nabla_{\mathrm{n}} \mathrm{u}_{\mathrm{k}}\right)=0$ leads after a few steps to the integral formula relating $\delta \sigma$ to $\delta u_{k}$ (sensitivity or derivative matrix):

$$
\int_{\Gamma} \delta \mathrm{u}_{\mathrm{k}} \sigma \nabla_{\mathrm{n}} \omega \mathrm{d} \Gamma=-\int_{\Omega} \delta \sigma\left(\nabla \omega \cdot \nabla \mathrm{u}_{\mathrm{k}}+\left(\frac{\mathrm{k} \pi}{\mathrm{a}}\right)^{2} \omega \mathrm{u}_{\mathrm{k}}\right) \mathrm{d} \Omega
$$




\section{REFERENCES}

[1] Butler, JE and Bonnecaze RT, "Imaging of particle Shear Migration with electrical Impedance Tomography, American Institute of Chemical Engineers Annual Meeting, Nov 1997.

[2] Ider, YZ, Gencer NG, Atalar E and Tosun H 1990 "Electrical impedance tomography of translationally uniform cylindrical objects with general cross-sectional boundaries ", IEEE Trans. Med. Imaging, Vol.9, no.1, pp. 49-59

[3] Lionheart, WRB "Problems of Shape and Dimension in EIT", ICEBI Barcelona 1998. 